\title{
DOSIMETRIC PREDICTORS OF RADIATION-INDUCED ACUTE NAUSEA AND VOMITING IN INTENSITY MODULATED RADIATION THERAPY ALONE FOR UNDIFFERENTIATED CARCINOMA OF THE NASOPHARYNX
}

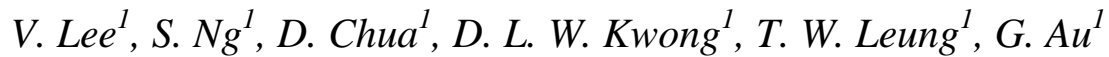 \\ ${ }^{1}$ THE UNIVERSITY OF HONG KONG, Clinical Oncology, Hong Kong, Hong \\ Kong (SAR) China
}

PURPOSE: To investigate dosimetric and clinical parameters that will predict radiationinduced acute nausea and vomiting in Intensity Modulated Radiation Therapy (IMRT) alone for undifferentiated carcinoma of the nasopharynx (NPC) MATERIALS: Thirty three consecutive patients with newly diagnosed NPC were treated with IMRT alone in this combined retrospective and prospective cohort. No chemotherapeutic agents were given as they either have stage I to IIB (AJCC 2002 classification) diseases or they were medically unfit for chemotherapy. Patients receiving any form of chemotherapy were excluded as cisplatin, the standard chemotherapeutic agent used in radical treatment of NPC is highly emetogenic which may make analysis difficult. Pre-radiotherapy magnetic resonance imaging (MRI) images of the head and neck region in the treatment position were co-registered with the planning computed tomography (CT) images of these patients immobilized in standard thermoplastic casts. The area postrema (AP) as well as the left and right vestibules (VB-L and VB-R respectively), which were well-recognized as the vomiting centres in the brain were contoured with the aid of the co-registered MRI images. A structure combining both VB-L and VB-R named as VB-T was also generated. They were all labeled as organs-at-risk (OAR). An additional $3 \mathrm{~mm}$ three-dimensional margin to these structures was created and labeled as AP+3mm, VB-L+3mm, VB$\mathrm{R}+3 \mathrm{~mm}$ and VB-T+3mm respectively to account for the physiological body motion and the setup error. No weightings were given to these structures during optimization in computer treatment planning. Dosimetric parameters including volumes of all OARs, minimum, mean, maximum, median, V30, V35, V40, V45, V50, V55, V60, V65, D05 and D01 were recorded from dose-volume histograms (DVH). Prospective monitoring of acute nausea and vomiting during IMRT was graded based on Common Toxicity Criteria Adverse Events Version 3.0 (CTCAE). A retrospective analysis of these dosimetric parameters together with other clinical parameters for their association with acute nausea and vomiting was performed using univariate and multivariate logistic regression. RESULTS: 5 (15.2\%) patients reported grade 1 and another $4(12.1 \%)$ patients reported grade 2 nausea respectively. Also 1 (3.0\%) patient complained grade 1 and 3 patients $(9.1 \%)$ experienced grade 2 vomiting. No patients developed protracted nausea and vomiting after completion of IMRT. For radiation-induced acute nausea, V40 of VB-L ( $\mathrm{p}=0.041), \mathrm{V} 35$ of VB-L+3mm ( $\mathrm{p}=0.050), \mathrm{V} 40$ of VB-L $+3 \mathrm{~mm}(\mathrm{p}=0.049)$, volume of VB-R $(p=0.034)$ and V40 of VB-T $(p=0.046)$ were found to be predictors on univariate analysis. On multivariate analysis, volume of VB-R $(\mathrm{p}=0.049)$ was the only predictor though V40 of VB-L ( $\mathrm{p}=0.076)$, V40 of VB-L+3mm ( $\mathrm{p}=0.071)$ and V40 of VBT $(\mathrm{p}=0.081)$ reached marginal significance. No parameters were predictors of radiationinduced acute vomiting since the number of patients and event outcomes were too small for analysis. No clinical parameters including sex, age, smoking history, overall stage, T- 
stage, N-stage were found to be predictors of both acute nausea and vomiting. CONCLUSIONS: Both vestibules deserve to be considered as essential OARs in an attempt to reduce radiation-induced acute nausea and vomiting. Weighting should be offered to these structures during optimization in the treatment planning of IMRT. Further patient accural is noteworthy to consolidate our preliminary findings. 